\title{
EMPREGO DE SÍLICA GEL ORGANICAMENTE MODIFICADA E IMPRESSA IONICAMENTE PARA PRÉ- CONCENTRAÇÃO SELETIVA ON-LINE DE ÍONS COBRE
}

\author{
Thiago Carvalho de Ávila, Mariana Gava Segatelli e Luiz Alberto Beijo \\ Departamento de Ciências Exatas, Universidade Federal de Alfenas, Rua Gabriel Monteiro da Silva, 714, 37130-000 Alfenas - \\ MG, Brasil \\ César Ricardo Teixeira Tarley* \\ Departamento de Ciências Exatas, Universidade Federal de Alfenas, Rua Gabriel Monteiro da Silva, 714, 37130-000 Alfenas - MG / \\ Departamento de Química Analítica, Instituto de Química, Universidade Estadual de Campinas, Cidade Universitária Zeferino \\ Vaz s/n, 13083-970 Campinas - SP, Brasil
}

Recebido em 16/2/09; aceito em 23/7/09; publicado na web em 8/1/10

\begin{abstract}
EMPLOY OF SILICA GEL ORGANICALLY MODIFIED AND IONICALLY IMPRINTED FOR SELECTIVE ON-LINE PRECONCENTRATION OF COPPER IONS. The present work purposes the preparation of a silica gel sorbent organically modified with 2-aminoethyl-3-aminobutylmethyldimethoxysilane (AAMDMS) and imprinted with $\mathrm{Cu}^{2+}$ ions by means surface imprinting technique and its use for selective on-line sorbent preconcentration of $\mathrm{Cu}^{2+}$ ions with further UV-VIS spectrophotometric determination by flow injection analysis. The $\mathrm{Cu}^{2+}$-imprinted silica gel, when compared with non imprinted silica gel and silica gel, showed from the binary mixture of $\mathrm{Cu}^{2+} / \mathrm{Ni}^{2+}$ relative selectivity coefficient $\left(k^{\prime}\right)$ of 6.84 and 5.43 and 6.64 and 19.83 for the mixture $\mathrm{Cu}^{2+} / \mathrm{Pb}^{2+}$, thus demonstrating higher selectivity of $\mathrm{Cu}^{2+}$-imprinted silica gel towards $\mathrm{Cu}^{2+}$ ions. Under optimized condition, the on-line preconcentration method provided detection limit of $3.4 \mu \mathrm{g} \mathrm{L} \mathrm{L}^{-1}$ and linear range ranging from 30.0 up to $300.0 \mu \mathrm{g} \mathrm{L} \mathrm{L}^{-1}(\mathrm{r}=0.995)$. The accuracy of method was successfully assessed by analyzing different kind of spiked water samples with recovery values ranging from 92.2 up to $103.0 \%$.
\end{abstract}

Keywords: imprinted silica gel; spectrophotometry; copper.

\section{INTRODUÇÃO}

Ao longo dos anos, observa-se uma crescente demanda por métodos analíticos visando a determinação de íons metálicos em baixas concentrações (em geral $\leq \mu \mathrm{g} \mathrm{L}^{-1}$ ) em uma variedade de amostras de interesse ambiental, clínico, alimentício etc. ${ }^{1}$ Apesar de existirem técnicas espectroanalíticas com seletividade e sensibilidade elevadas, como a espectrometria de massas com plasma acoplado indutivamente (ICP-MS), seus altos custos de aquisição e manutenção ainda as tornam indisponíveis em muitos laboratórios de análise química. Técnicas mais acessíveis, incluindo a espectrometria de absorção atômica em chama (FAAS) e a espectrofotometria UV/Vis, não apresentam, por outro lado, sensibilidade analítica suficiente para a determinação de íons metálicos em baixas concentrações. ${ }^{2}$

Considerando as técnicas supracitadas, problemas associados à baixa sensibilidade inviabilizam a análise direta da amostra em sua forma original, tendo em vista os baixos níveis dos analitos na presença de altas concentrações de concomitantes, da ordem de $\mathrm{g} \mathrm{L}^{-1}$. Adicionalmente, mesmo com o uso de técnicas sensíveis, a incompatibilidade da amostra com o equipamento pode inviabilizar a análise. Assim sendo, tratamentos prévios da amostra baseados em etapas de extração e/ou pré-concentração visando eliminar o interferente e/ou pré-concentrar o analito ainda continuam sendo alvo de estudos. ${ }^{3}$

As modalidades mais usuais de pré-concentração podem ser classificadas em: coprecipitação, precipitação, pré-concentração eletroquímica, extração líquido-líquido (ELL) (envolvendo a extração por ponto nuvem e membranas líquidas) e extração em fase sólida (SPE). ${ }^{4}$ Destes, os procedimentos baseados em SPE sofreram os mais rápidos desenvolvimentos, por conta da simplicidade, elevados fatores de pré-concentração, fácil regeneração da fase sólida, facilidade de automação, custos reduzidos em função do baixo consumo de reagen-

*e-mail: ctarleyquim@yahoo.com.br tes e, principalmente, devido à existência de diferentes adsorventes sólidos. Basicamente, os adsorventes são classificados como sólidos polares, apolares, resinas quelantes e resinas trocadoras de íons, onde os mais comuns são: sílica modificada $\left(\mathrm{C}_{18}\right)$, polímeros adsorventes tipo Amberlite IRA-904, PTFE (politetrafluoroetileno), carvão ativado, espuma de poliuretano, alumina, sílica gel etc. ${ }^{5}$

De maneira geral, a grande desvantagem destes tipos de adsorventes é a baixa seletividade. Devido à complexidade de algumas matrizes, aos baixos níveis dos constituintes de interesse e à presença de interferentes em potencial, a etapa de extração/pré-concentração pode apresentar baixo desempenho. Situações desta natureza podem ser contornadas fazendo-se uso de adsorventes sólidos seletivos baseados no conceito de impressão química. ${ }^{6}$

A técnica de impressão química, inicialmente desenvolvida para moléculas e, posteriormente, para íons metálicos, consiste em uma reação de polimerização, na qual o monômero interage por meio de ligação covalente ou não com o template, formando um arranjo tridimencional. ${ }^{7,8}$ Após a remoção do template, no material outrora preparado, cavidades seletivas em forma e tamanho ao analito são geradas. Polímeros impressos com íons (IIPs - Ion Imprinted Polymers) representam uma particularidade daqueles impressos com moléculas e possuem as mesmas vantagem, tais como seletividade, simplicidade e conveniência na síntese. ${ }^{9}$ Nestes polímeros, a seletividade é governada pela especificidade do ligante (monômero) em relação ao íon, pela geometria de coordenação do complexo, pelo número de coordenação dos íons e, também, pelo tamanho e carga do íon. ${ }^{10}$ Os IIPs são, em sua grande maioria, preparados pelo método em bulk em sistema homogêneo, basicamente devido à sua simplicidade. Todavia, estes polímeros exibem partículas com tamanhos irregulares, incompleta remoção do template e pobre acessibilidade dos analitos à cavidade seletiva. ${ }^{11}$ Desta forma, recentemente métodos promissores de impressão bidimencional em superfície de suportes sólidos têm atraído bastante atenção, uma vez que o template pode 
ser completamente removido do adsorvente e, como consequência, tornando as cavidades seletivas presentes na superfície mais acessíveis à retenção do analito. A sílica gel é considerada um suporte sólido ideal para impressão bidimencional, pois apresenta estabilidade química em condições ácidas e não sofre inchamento sob vazões elevadas. Ordinariadamente, adsorventes com impressão bidimencional são preparados pelo método heterogêneo, que consiste em modificar organicamente a superfície da sílica gel com precursores organossilanos altamente reativos com a superfície da sílica e que contém usualmente átomos de nitrogênio ou enxofre. A interação destes átomos com o íon metálico é responsável pela formação da cavidade seletiva no adsorvente. ${ }^{12}$ Cabe ressaltar que a modificação da superfície da sílica gel com precursores organossilanos também confere, sobretudo, maior estabilidade química à sílica. $\mathrm{O}$ primeiro trabalho envolvendo o uso adsorventes impressos com íons em superfície de sílica gel organicamente modificada para extração seletiva de íon é bastante recente, datado de $2006,{ }^{13}$ para os íons $\mathrm{Ni}^{2+}$. Posteriormente, outros trabalhos foram desenvolvidos para os íons $\mathrm{Hg}^{2+}, \mathrm{Zr}^{4+}, \mathrm{Cr}^{3+}$, $\mathrm{Dy}^{3+}, \mathrm{Pd}^{2+}, \mathrm{Fe}^{3+}$ e $\mathrm{Zn}^{2+} .{ }^{14-20}$ É importante salientar que nestes métodos a determinação dos íons metálicos foi feita por técnicas naturalmente seletivas e de custos elevados, como a espectrometria de emissão atômica com plasma acoplado indutivamente (ICP OES) e ICP-MS, não refletindo fidedignamente o desempenho seletivo adsorvente em amostras reais. Nos procedimentos de extração não foram adotados, ainda, sistemas de análise por injeção em fluxo, substancialmente superiores aos sistemas em batelada.

Assim, o objetivo do presente estudo foi avaliar o desempenho seletivo da sílica organicamente modificada com o precursor funcional 2-aminoetil-3-aminobutilmetildimetoxissilano e impressa ionicamente para pré-concentração on-line de íons cobre com determinação por espectrofotometria UV/vis. Os íons cobre previamente pré-concentrados foram determinados em $452 \mathrm{~nm}$ após complexação com dietilditiocarbamato (DDTC). Adotou-se este complexante não específico para os íons cobre com intuito de que a seletividade do método fosse atribuída apenas ao adsorvente impresso.

\section{PARTE EXPERIMENTAL}

\section{Instrumentação}

As medidas espectrofotométricas empregando sistema de análise por injeção em fluxo (FIA) foram feitas em um espectrofotômetro de feixe simples Femto, modelo 435 (São Paulo-Brasil) equipado com uma cela de fluxo de $1,0 \mathrm{~cm}$ de caminho óptico. A aquisição de dados foi realizada por meio de uma interface PCL 711 com programação em macro Visual Basic (Microsoft Excel). Para propulsão dos padrões, amostras e reagentes foi empregada uma bomba peristáltica Ismatec (Zurique-Suíça) de 8 canais contendo tubos de Tygon de diferentes diâmetros e tubos de polietileno com $0,8 \mathrm{~mm}$ de diâmetro interno. Um pHmetro de bancada [Metrohm 827 pH lab (São Paulo, Brasil)] foi utilizado para as aferições de $\mathrm{pH}$. Um injetor proporcional de três peças ${ }^{21}$ feito em Teflon ${ }^{\circledR}$ foi utilizado para introdução dos padrões e amostras no sistema FIA.

Para os estudos de seletividade e efeito de impressão no adsorvente, foi utilizado um espectrômetro de absorção atômica com atomização em chama (FAAS) Shimadzu, modelo AA-6800 equipado com lâmpada de cátodo oco e deutério para correção de fundo.

A morfologia dos materiais foi avaliada por microscopia eletrônica de varredura (MEV) usando-se um microscópio de varredura eletrônica, modelo JEOL JSM-6360 LV (Tokyo, Japão) com voltagem de aceleração de $20 \mathrm{kV}$.

Para o preparo das amostras, foi efetuado o recobrimento das mesmas com uma liga de $\mathrm{Au} / \mathrm{Pb}$ em um equipamento Bal-Tec MED 020.
A análise térmica dos adsorventes foi realizada em um analisador termogravimétrico (TA Instruments, modelo TGA 2950, California, USA), onde aproximadamente $10 \mathrm{mg}$ de amostras foram aquecidas de 30 a $980{ }^{\circ} \mathrm{C}$, numa taxa de aquecimento de $10{ }^{\circ} \mathrm{C} \mathrm{min}{ }^{-1}$, sob atmosfera de argônio.

Os espectros na região do infravermelho foram obtidos por meio de um espectrômetro de infravermelho (Bomem Michelson MB102). Os experimentos foram realizados usando pastilhas de $\mathrm{KBr}$ e faixa espectral de 4000 a $400 \mathrm{~cm}^{-1}$. As isotermas de adsorção foram processadas no programa computacional STATÍSTICA (versão 6.0).

\section{Reagentes e soluções}

A sílica gel 60, usualmente empregada para cromatografia de camada delgada (Merck, 70-230 mesh), foi ativada com HCl 6 mol $\mathrm{L}^{-1} \mathrm{em}$ refluxo por $8 \mathrm{~h}$. O precursor funcional 2-aminoetil-3-aminobutilmetildimetoxissilano (Fluka) foi usado sem prévia purificação.

Todas as soluções foram preparadas empregando-se água deionizada proveniente de um sistema de purificação de água Milli- $\mathrm{Q}^{\circledR}$ da Millipore ${ }^{\circledR}$ (Bedford, MA, USA) e todos os reagentes usados foram de grau analítico. A fim de minimizar contaminações das amostras, todas as vidrarias foram mantidas em solução de $\mathrm{HNO}_{3} 10 \%$ (v/v) durante $24 \mathrm{~h}$. A solução estoque de DDTC de 2,0\% (m/v) foi preparada pela dissolução de 2,0 g de dietilditilcarbamato de sódio (Merck) em 20,0 $\mathrm{mL}$ de álcool etílico (Merck) e água quente, cujo volume foi completado em balão volumétrico de 100,0 mL. A solução foi armazenada em refrigerador e diluições apropriadas foram realizadas em água deionizada.

\section{Síntese da sílica gel organicamente modificada e impressa ionicamente (SGII)}

A sílica gel $\left(\mathrm{SiO}_{2}\right)$ é um óxido inorgânico, no qual cada átomo de silício é coordenado tetraedricamente com quatro átomos de oxigênio formando uma estrutura tridimensional por meio de grupos siloxano ( $\mathrm{Si}-\mathrm{O}-\mathrm{Si}$ ).

Os grupos silanois ( $\mathrm{Si}-\mathrm{OH})$ em sua superfície permitem a imobilização de uma grande variedade de moléculas organofuncionais. Porém, a sílica gel comercial apresenta baixa concentração dos grupos silanois na superfície, o que torna necessária a ativação de sua superfície. A ativação foi realizada utilizando-se $3,0 \mathrm{~g}$ de sílica gel, mantida em refluxo durante $8 \mathrm{~h}$ em $\mathrm{HCl} \mathrm{6,0} \mathrm{mol} \mathrm{L}^{-1}$. Em seguida, a sílica foi filtrada a vácuo e mantida em estufa durante $20 \mathrm{~h}$ à 80 ${ }^{\circ} \mathrm{C} .{ }^{19}$ Após esta etapa, deu-se início ao procedimento de impressão iônica que consiste em misturar $1,0 \mathrm{~g}$ de $\mathrm{CuCl}_{2} \cdot\left(\mathrm{H}_{2} \mathrm{O}\right)_{2}$ previamente dissolvido em 30,0 mL de metanol com 3,0 mL de 2-aminoetil-3aminobutilmetildimetoxissilano. A mistura reacional foi mantida em refluxo por $1 \mathrm{~h}$. Este procedimento foi feito a fim de promover a complexação dos íons cobre com o grupo diamina presente no precursor funcional 2-aminoetil-3-aminobutilmetildimetoxissilano. Posteriormente, $3,0 \mathrm{~g}$ de sílica gel ativada foram adicionadas à solução e mantida em refluxo por mais $20 \mathrm{~h} .{ }^{17,18}$ Finalmente, o material foi filtrado a vácuo, lavado com $70,0 \mathrm{~mL}$ de $\mathrm{HCl}$ 6,0 $\mathrm{mol} \mathrm{L}^{-1}$ para remoção dos íons cobre e mantido em estufa por $12 \mathrm{~h}$ à $80^{\circ} \mathrm{C}$. A completa remoção dos íons cobre foi monitorada por FAAS. A fim de avaliar o efeito de impressão dos íons cobre na sílica modificada, efetuou-se a mesma síntese supracitada, porém sem a adição do cloreto de cobre. Este material foi designado como sílica gel sem impressão (SGSI).

\section{Sistema de pré-concentração em fluxo}

A Figura 1 mostra o diagrama do sistema de pré-concentração on-line. No sistema operado com o injetor na posição amostragem (Figura 1a), 14,0 $\mathrm{mL}$ de solução de íons $\mathrm{Cu}^{2+}$ tamponada (pH 8,35 
em tampão $\mathrm{NH}_{3} / \mathrm{NH}_{4}^{+} 0,05$ mol L-1) são percolados através de uma minicoluna de PVC (cloreto de polivinila) contendo $100 \mathrm{mg}$ de SGII a uma vazão de $10,2 \mathrm{~mL}$ min $^{-1}$. Uma pequena quantidade de lã de vidro foi colocada em ambos os lados da minicoluna, a fim de impedir a perda de SGII durante as etapas de pré-concentração/eluição. O eluente $\left(\mathrm{HNO}_{3} 0,5 \mathrm{~mol} \mathrm{~L}^{-1}\right)$, bombeado em direção ao detector a

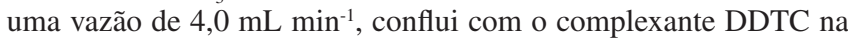
concentração de $0,01 \%(\mathrm{~m} / \mathrm{V})$. Após esta etapa, a eluição é acionada pela alteração da posição do injetor, onde a minicoluna é posicionada no fluxo do eluente permitindo a dessorção em contracorrente dos íons $\mathrm{Cu}^{2+}$. Os íons $\mathrm{Cu}^{2+}$ dessorvidos confluem com a solução do agente complexante DDTC numa vazão de $3,0 \mathrm{~mL} \mathrm{~min}^{-1}$. A formação do complexo $\mathrm{Cu}$ (DDTC), se completa através da bobina reacional de $94 \mathrm{~cm}$ com posterior detecção espectrofotométrica em $452 \mathrm{~nm}$.

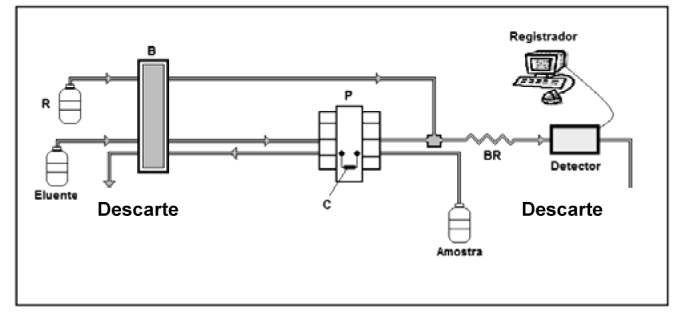

(a)

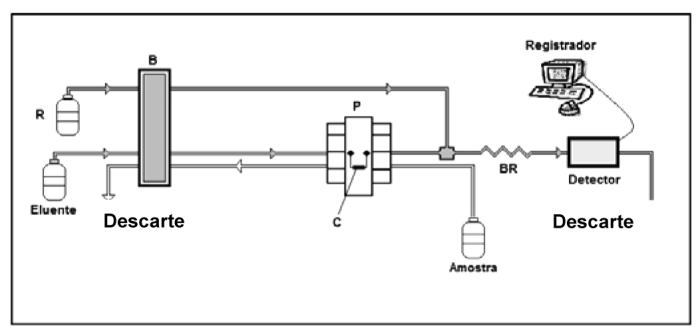

(b)

Figura 1. Diagrama do sistema de pré-concentração utilizado para deter-

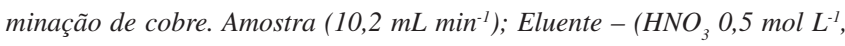

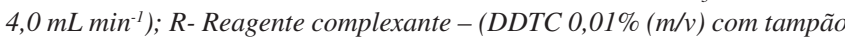
$\mathrm{NH}_{3} / \mathrm{NH}_{4}^{+}$0,05 mol $\left.L^{-1}\right) ; B$ - Bomba Peristáltica; $P$ - injetor proporcional; C-minicoluna de PVC; Detector; Registrador; BR - Bobina Reacional de $94 \mathrm{~cm}$, (a) etapa de pré-concentração; (b) etapa de eluição

\section{Construção da isoterma de adsorção}

Com intuito de se obter a capacidade máxima adsortiva (CMA) da SGII frente os íons $\mathrm{Cu}^{2+}$ foi construída uma isoterma de adsorção, cujo ajuste dos dados experimentais foi avaliado por meio de isotermas lineares de Langmuir e Freundlich, bem como por isotermas não lineares de Langmuir, Freundlich e Langmuir-Freundlich.

Nos experimentos, 50,0 mg de SGII foram agitados por $15 \mathrm{~min}$ em frascos fechados de polietileno à temperatura ambiente com 20,0 $\mathrm{mL}$ de solução de $\mathrm{Cu}^{2+}$, em concentrações crescentes de 1,0 a 50,0 $\mathrm{mg} \mathrm{L}^{-1}$. As soluções foram mantidas em $\mathrm{pH} 8,35$ com tampão $\mathrm{NH}_{3} /$ $\mathrm{NH}_{4}^{+}$0,05 mol L-1. Após o tempo de agitação, o sobrenadante foi analisado por FAAS. A quantidade de íons $\mathrm{Cu}^{2+}$ adsorvida na SGII foi determinada através da diferença entre a concentração inicial e a concentração de equilíbrio no sobrenadante.

\section{Avaliação do efeito de impressão na SGII}

Para avaliar o efeito de impressão na SGII, ou seja, a seletividade para os íons $\mathrm{Cu}^{2+}$, foram realizados estudos de adsorção de soluções binárias de $\mathrm{Cu}^{2+} / \mathrm{Ni}^{2+}$ ou $\mathrm{Cu}^{2+} / \mathrm{Pb}^{2+}$ em SGII, SGSI, bem como em sílica

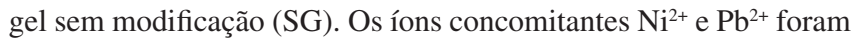
escolhidos neste estudo por serem divalentes, igualmente ao $\mathrm{Cu}^{2+}$, e por formarem complexos estáveis com o grupo diamina presente no precursor funcional. Por conta destes fatores, uma maior afinidade dos íons $\mathrm{Cu}^{2+}$ pela SGII representa um forte indicativo da impressão iônica criada no adsorvente.

Os experimentos foram realizados empregando minicolunas de PVC preenchidas com 100,0 mg de SGII, SGSI ou SG e percoladas com $14 \mathrm{~mL}$ de solução binária de $\mathrm{Cu}^{2+} / \mathrm{Ni}^{2+}$ ou $\mathrm{Cu}^{2+} / \mathrm{Pb}^{2+}$, nas respectivas concentrações de 2,0 e 4,0 $\mathrm{mg} \mathrm{L}^{-1}$ para cada íon metálico. $\mathrm{O}$ pH das soluções foi mantido em 8,35 em tampão $\mathrm{NH}_{3} / \mathrm{NH}_{4}{ }^{+} 0,05 \mathrm{~mol} \mathrm{~L}{ }^{-1}$. As quantidades dos íons metálicos presentes no efluente da minicoluna foram determinadas por FAAS. Mediante estes experimentos, foram determinados os parâmetros relacionados ao desempenho seletivo dos adsorventes incluindo o coeficiente de distribuição $\left(\mathrm{K}_{\mathrm{d}}\right)$, coeficiente de seletividade $(k)$ e coeficiente de seletividade relativo $\left(k\right.$ '). Os coeficientes de distribuição dos íons $\mathrm{Cu}^{2+}, \mathrm{Ni}^{2+} \mathrm{e} \mathrm{Pb}^{2+}$ foram calculados conforme a Equação 1:

$\mathrm{K}_{\mathrm{d}}=\left[\frac{\left(\mathrm{C}_{\mathrm{i}}-\mathrm{C}_{\mathrm{f}}\right)}{\mathrm{C}_{\mathrm{f}}}\right] \mathrm{x} \frac{\mathrm{V}_{\mathrm{s}}(\mathrm{mL})}{\text { massa do adsorvente }(\mathrm{g})}$

onde $\mathrm{K}_{\mathrm{d}}, \mathrm{C}_{\mathrm{i}}, \mathrm{C}_{\mathrm{f}}$ e Vs representam o coeficiente de distribuição, a concentração inicial da solução pré-concentrada, a concentração final ou a concentração do eluato e volume de solução pré-concentrada, respectivamente. $\mathrm{O}$ coeficiente de seletividade $(k)$ é definido como a razão entre o coeficiente de distribuição dos íons $\mathrm{Cu}^{2+}$ e o coeficiente de distribuição da espécie concomitante, $\mathrm{Ni}^{2+} \mathrm{Ou} \mathrm{Pb}^{2+}$, para um mesmo adsorvente.

Com o intuito de comparar os valores de $(k)$ obtidos para SGII com aqueles obtidos para SGSI e SG empregou-se a Equação 2, que permite estimar o efeito de impressão na SGII.

$k^{\prime}=\frac{k_{\mathrm{SGII}}}{k_{\text {controle (SGSI ou SG) }}}$

onde $k$ ' é coeficiente de seletividade relativo, $k_{\mathrm{SGII}}$ é o coeficiente de seletividade da SGII e $k_{\text {controle }}$ é o coeficiente de seletividade da SGSI ou SG. ${ }^{22}$

\section{RESULTADOS E DISCUSSÃO}

\section{Caracterização dos adsorventes}

Os resultados provenientes dos espectros de FT-IR mostraram bandas relativas ao esqueleto da sílica presentes em $3432 \mathrm{~cm}^{-1}$ devido à vibração $\mathrm{Si}-\mathrm{OH}$, e em 1232 e $1097 \mathrm{~cm}^{-1}$ relativas aos estiramentos assimétricos dos grupos Si-O-Si. Também, uma banda larga entre 3750 a $3100 \mathrm{~cm}^{-1}$ foi atribuída à água remanescente adsorvida. A absorção em $1629 \mathrm{~cm}^{-1}$ é referente às vibrações angulares das moléculas de água. Para a SGII e SGSI observou-se, respectivamente, o surgimento de bandas de absorção pouco intensas em 2971 e $2964 \mathrm{~cm}^{-1}$, referentes à vibração de estiramento assimétrico do grupo $(\mathrm{C}-\mathrm{H})$, indicando a presença de 2-aminoetil-3-aminobutilmetildimetoxissilano ancorado na superfície. A banda em $959 \mathrm{~cm}^{-1}$ é atribuída ao estiramento simétrico dos grupos siloxanos ( $v_{\text {sim }}$ Si-O-Si), ao passo que em 806 e 467 $\mathrm{cm}^{-1}$ as bandas referem-se à vibração de O-Si-O. ${ }^{22,24}$

A estabilidade térmica dos adsorventes foi determinada por análise termogravimétrica. De acordo com a Figura 2, a sílica gel perde cerca de $7 \%$ em massa com o aquecimento de 25 a $112{ }^{\circ} \mathrm{C}$ atribuída à água adsorvida fisicamente. Posteriormente, com o aquecimento de 112 a $1015{ }^{\circ} \mathrm{C}$ há um decréscimo de $3 \%$ em massa, por conta da 
condensação dos grupos silanois ligados na sílica. Com relação à sílica funcionalizada, também se constata a perda de massa referente à água adsorvida fisicamente, porém com 1,5 e 5,5\%, respectivamente, para a SGSI e SGII. O acréscimo da temperatura de 118 a $324{ }^{\circ} \mathrm{C}$ confere perda de massa de $2 \%$ para SGSI e de $6 \%$ para SGII, a qual é atribuída à decomposição da fração orgânica do 2-aminoetil-3aminobutilmetildimetoxissilano, corroborando com os espectros de infravermelho. ${ }^{25}$ Sob temperaturas superiores a $324{ }^{\circ} \mathrm{C}$ ocorre perda de água resultante da condensação dos grupos silanois.

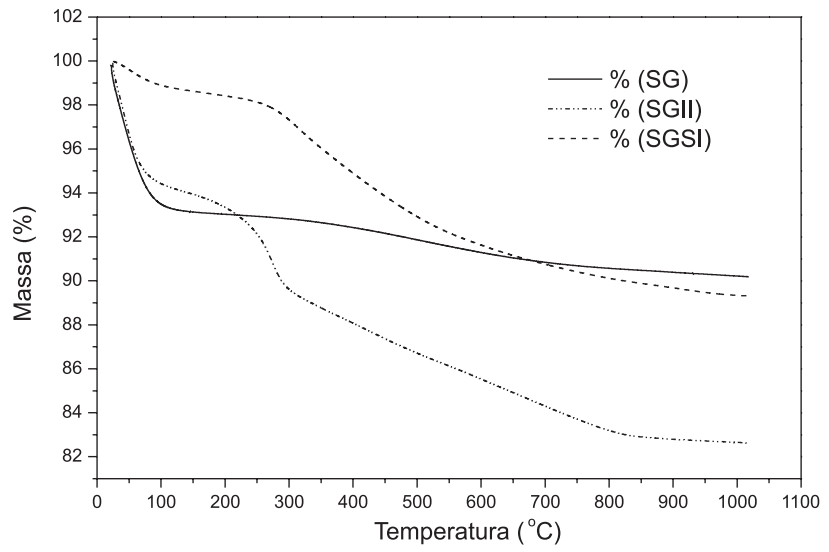

Figura 2. Curvas termogravimétricas dos adsorventes SGII, SGSI e SG

No que concerne às micrografias mostradas na Figura 3, verificase que as partículas de sílica não são esféricas, porém esta característica não reflete um baixo desempenho do sistema de extração em fase sólida, tendo em vista as baixas vazões usualmente empregadas em sistemas em fluxo. Também, cabe salientar que a SGII possui superfície ligeiramente mais porosa que a SG e a SGSI, condições propícias em processos adsortivos.
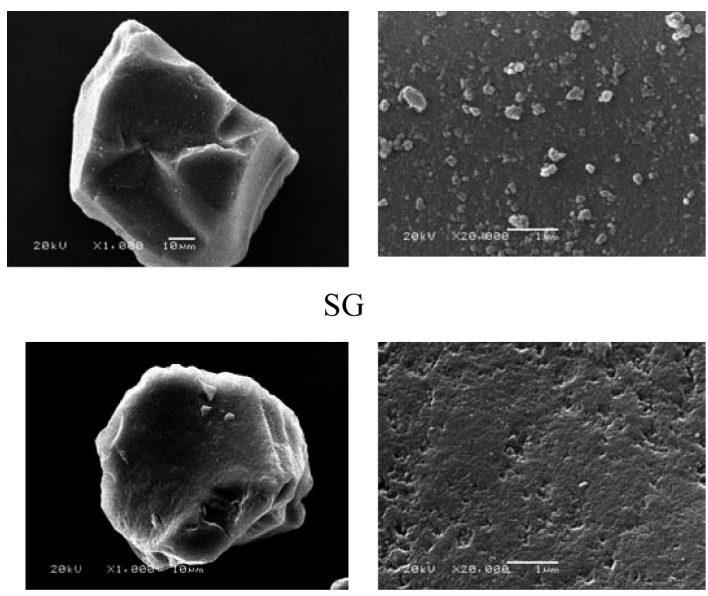

SGII
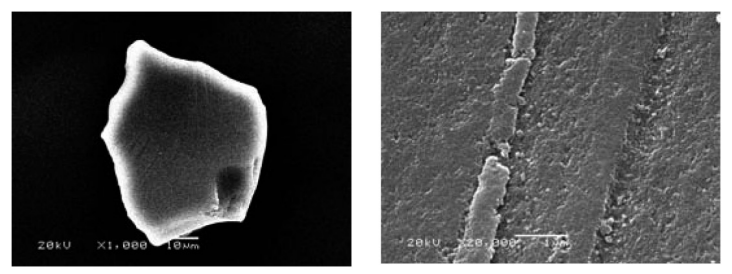

SGSI

Figura 3. Micrografia eletrônica de varredura dos adsorventes $S G$, SGII, e SGSI com ampliação de 20.000 e 1.000 vezes

\section{Otimização do sistema de pré-concentração em fluxo}

\section{Efeito do pH, tipo e concentração do tampão}

As variáveis químicas e de fluxo foram otimizadas a fim de se obter a melhor sensibilidade analítica. Primeiramente, foi investigado o efeito do $\mathrm{pH}$ de uma solução de $\mathrm{Cu}^{2+}$ de $250 \mu \mathrm{g} \mathrm{L}^{-1}$ na adsorção em SGII. Estes experimentos foram realizados com as seguintes variáveis fixadas: volume e vazão de pré-concentração de $14,0 \mathrm{~mL}$ e 5,0 mL $\min ^{-1}$, respectivamente; concentração e vazão do eluente $\left(\mathrm{HNO}_{3}\right)$ em 1,0 mol L-1 e 4,0 mL min ${ }^{-1}$, respectivamente; e concentração do DDTC em 0,5\% (m/v) e vazão de 3,0 $\mathrm{mL} \mathrm{min}^{-1}$.

Como pode ser observado na Figura 4, os sinais analíticos para valores de $\mathrm{pH}$ variando de 6 a 8 são consideravelmente inferiores àqueles em $\mathrm{pH}$ acima de 8,35. Este comportamento é atribuído à protonação dos átomos de nitrogênio do grupo diamina, resultando em repulsão eletrostática com os íons $\mathrm{Cu}^{2+}$.

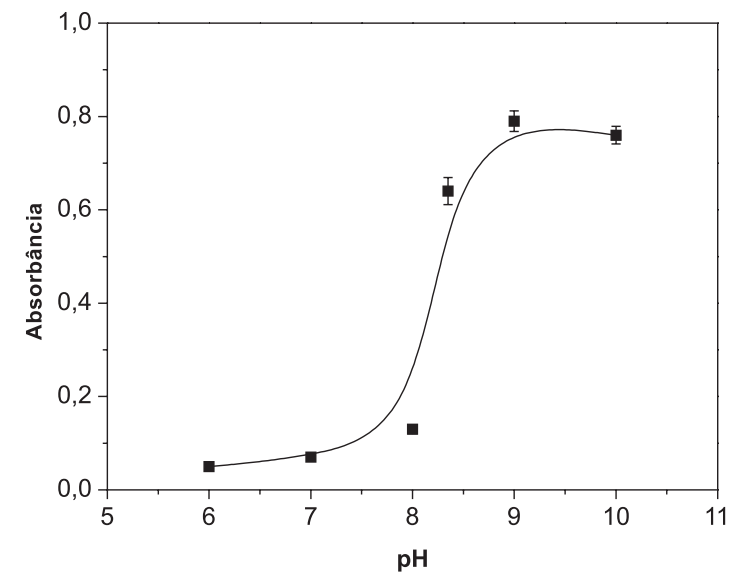

Figura 4. Efeito do pH da amostra na pré-concentração de $250 \mu \mathrm{g} L^{-1}$ de $\mathrm{Cu}^{2+}$ em 100 mg de SGII. Condições: volume e vazão de pré-concentração de $14 \mathrm{~mL}$ e 5,0 $\mathrm{mL} \mathrm{min}^{-1}$, respectivamente; concentração e vazão do eluente

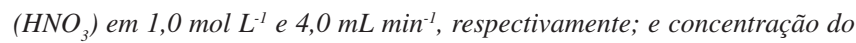
DDTC em $0,5 \%(\mathrm{~m} / \mathrm{v})$ e vazão de $3,0 \mathrm{~mL} \mathrm{~min}^{-1}$

Foram realizados estudos em condições de maior acidez ( $\mathrm{pH}$ abaixo de 6), porém os sinais analíticos foram substancialmente baixos. Cabe salientar que, para os valores de $\mathrm{pH}$ de 6 a 8, os padrões foram tamponados com tampão fosfato, ao passo que para valores de $\mathrm{pH} 8,35$ a 10 se empregou tampão borato, ambos na concentração de $0,1 \mathrm{~mol}$ $\mathrm{L}^{-1}$. Considerando que os melhores resultados foram obtidos na faixa de pH 8,35 a 10, avaliou-se o efeito do tipo de tampão neste intervalo.

Constatou-se que o uso de tampão amoniacal em todo o intervalo estudado de $\mathrm{pH}(8,35 \mathrm{a} 10)$ apresenta resultados melhores em detrimento ao uso de tampão borato. Assim sendo, fixou-se o tampão amoniacal em $\mathrm{pH}$ em 8,35 visando preservar a estabilidade química, bem como o tempo de vida da SGII. O efeito da concentração do tampão $\mathrm{NH}_{3} / \mathrm{NH}_{4}{ }^{+}$foi investigado de 0,005 a $0,3 \mathrm{~mol} \mathrm{~L}^{-1}$. Observou-se que os sinais analíticos foram significativamente crescentes com o aumento da concentração até $0,05 \mathrm{~mol} \mathrm{~L}^{-1}$, enquanto que o emprego de concentrações maiores não resulta em ganhos significativos de sinal analítico. Assim, a concentração do tampão $\mathrm{NH}_{3} / \mathrm{NH}_{4}^{+}$na amostra foi mantida em $0,05 \mathrm{~mol} \mathrm{~L}^{-1}$.

\section{Efeito da vazão de pré-concentração}

Nos estudos dedicados ao efeito da vazão de pré-concentração, observou-se que os sinais analíticos não diferiram significativamente para vazões variando de 1,6 a 10,2 $\mathrm{mL} \mathrm{min}^{-1}$. Este comportamento mostra que a cinética de adsorção dos íons $\mathrm{Cu}^{2+}$ sobre a SGII é bastante rápida. Do ponto vista prático, é possível obter alta sensibilidade 
empregando elevados volumes de pré-concentração, sem resultar em acentuado decréscimo na frequência analítica.

\section{Efeito da concentração e do tipo de eluente}

Foram testados como eluentes os ácidos $\mathrm{HCl}$ e $\mathrm{HNO}_{3}$, ambos na concentração de $1,0 \mathrm{~mol} \mathrm{~L}^{-1}$. O decréscimo da resposta analítica (cerca de 60\%) quando se emprega $\mathrm{HCl}$ em relação ao uso de $\mathrm{HNO}_{3}$ ocorre, possivelmente porque os íons cloreto, naturalmente ligantes mais fortes que os íons nitrato, formam cloro-complexos com $\mathrm{Cu}^{2+}$ impedindo parcialmente a complexação do analito com DDTC. Desta forma, decidiu-se utilizar $\mathrm{HNO}_{3}$ como eluente. As concentrações deste ácido foram investigadas dentro da faixa de 0,10 a $1,30 \mathrm{~mol} \mathrm{~L}^{-1}$. Em concentrações menores (abaixo de $0,5 \mathrm{~mol} \mathrm{~L}^{-1}$ ), nota-se que a quantidade de ácido é insuficiente para eluir quantitativamente os íons $\mathrm{Cu}^{2+}$, resultando em efeito de memória a cada ciclo de pré-concentração/ eluição. Considerando-se este resultado, adotou-se a concentração de $0,5 \mathrm{~mol} \mathrm{~L}^{-1}$ para o $\mathrm{HNO}_{3}$ para os estudos subsequentes.

\section{Efeito da concentração do complexante (DDTC)}

O efeito da concentração do complexante (DDTC) no sinal analítico foi investigado dentro da faixa de 0,01 a $0,75 \%(\mathrm{~m} / \mathrm{v})$. Os resultados mostram que a absorbância obtida na concentração de $0,01 \%$ (m/v) é $16 \%$ maior que aquela obtida para a concentração de $0,75 \%(\mathrm{~m} / \mathrm{v})$. Em todas as condições estudadas, há um excesso do número de mols do DDTC em relação aos íons $\mathrm{Cu}^{2+}$, porém, a concentração excessiva de DDTC no meio pode reduzir a absortividade molar do complexo $\mathrm{Cu}(\text { DDTC })_{2}{ }^{26}$

Mediante a otimização da concentração de DDTC em $0,01 \%$ (m/v), avaliou-se o efeito do $\mathrm{pH}$ da solução de DDTC tamponada ( $\mathrm{pH} 7$ a 10). Não foi observada nenhuma eficácia no tamponamento da solução de DDTC em diferentes valores de $\mathrm{pH}$, pois os sinais obtidos foram equivalentes àqueles obtidos quando a solução de DDTC foi empregada sem prévio tamponamento. Este resultado revela que a complexação do DDTC com os íons $\mathrm{Cu}^{2+}$ também ocorre em meio fortemente ácido.

\section{Construção da isoterma de adsorção}

Isotermas de adsorção de íons metálicos em adsorventes sólidos devem ser construídas sob condições previamente otimizadas, i.e, $\mathrm{pH}$ de adsorção, tempo de equilíbrio entre o adsorvato e o adsorvente e temperatura constante. Estudos realizados a fim de se obter o tempo de equilíbrio foram conduzidos sob temperatura ambiente e nas condições de $\mathrm{pH}$ adotadas no sistema em fluxo, ou seja, solução em pH 8,35 em tampão $\mathrm{NH}_{3} / \mathrm{NH}_{4}^{+} 0,05 \mathrm{~mol} \mathrm{~L}^{-1}$. Foram empregados $20,0 \mathrm{~mL}$ de solução de $\mathrm{Cu}^{2+}$ na concentração de $2,0 \mathrm{mg} \mathrm{L}^{-1}$. Constatou-se que o tempo de agitação exibe influência no percentual de adsorção dos íons $\mathrm{Cu}^{2+}$ na SGII, sendo que $15 \mathrm{~min}$ foram suficientes para atingir o tempo de equilíbrio.

A isoterma de adsorção de $\mathrm{Cu}^{2+}$ em SGII construída com os dados experimentais é apresentada na Figura 5. Segundo classificação de Gilles a isoterma é do tipo L (tipo 4), ou seja, côncava seguida frequentemente por um ponto de inflexão. ${ }^{27}$ Os modelos de adsorção normalmente fazem uso de equações linearizáveis, as quais permitem facilmente estimar os parâmetros graficamente. ${ }^{28}$ Porém, alguns modelos linearizados de isotermas, apesar de apresentarem ajuste dos dados experimentais, proporcionaram erros muito grandes nos valores da capacidade máxima adsortiva, por conta de não considerarem desvios sistemáticos da isoterma ajustada. A linearização da isoterma de adsorção revelou que o modelo linear de Langmuir não reproduz os dados experimentais $\left(\mathrm{R}^{2}=0,3224\right)$, ao passo que a linearização segundo Freundlich representa ajuste um tanto quanto adequado aos dados $\left(\mathrm{R}^{2}=0,9488\right)$. Todavia, como pode ser observado na Figura 5 e Tabela 1, o modelo não linear de Lagmuir-Freundlich é o que melhor se ajusta aos dados experimentais, com coeficiente de determinação de $97,91 \%$ e baixo erro padrão em relação aos parâmetros $\mathrm{M}$ (capacidade máxima de adsorção em $\mathrm{mg} \mathrm{g}^{-1}$ ), $\mathrm{K}$ (parâmetro de afinidade) e $\beta$ (parâmetro empírico que varia com o grau de heterogeneidade da superfície do adsorvente), apresentando uma capacidade máxima adsortiva de 14,66 $\mathrm{mg} \mathrm{g}^{-1}$ dos íons $\mathrm{Cu}^{2+}$ na SGII. Este valor é superior em comparação a alguns adsorventes poliméricos impressos com íons $\mathrm{Cu}^{2+} \cdot{ }^{29,30}$ Nota-se que o modelo de Langmuir não linear sobre-estima a capacidade máxima adsortiva $\left(26,85 \mathrm{mg} \mathrm{g}^{-1}\right)$ dos íons $\mathrm{Cu}^{2+}$ na SGII, se diferenciando sobremaneira dos dados experimentais.

Do ponto de vista físico-químico pode-se tecer alguns comentários para justificar o melhor ajuste do modelo não linear de LagmuirFreundlich. A superfície da SGII contém dois sítios adsortivos; um não específico representado pelos grupos silanois e outro, específico, representado pelo grupo diamina do precursor funcional 2-aminoetil3-aminobutilmetildimetoxissilano. O comportamento adsortivo destes materiais, ou seja, que contêm sítios heterogêneos tem sido modelado com acurácia pela isoterma não linear de Lagmuir-Freundlich. ${ }^{31}$

\section{Avaliação do efeito de impressão na SGII e teste de interferência}

Na Tabela 2 estão apresentados os valores de $\mathrm{K}_{\mathrm{d}}$ para $\mathrm{Cu}^{2+}, \mathrm{Ni}^{2+} \mathrm{e}$ $\mathrm{Pb}^{2+}$ nos materiais SGII, SGSI e SG, além dos valores do coeficiente

Tabela 1. Equações das isotermas de adsorção e estimativas dos parâmetros das isotermas ajustadas com respectivo erro padrão (EP) e coeficiente de determinação $\mathrm{R}^{2}$

\begin{tabular}{|c|c|c|c|c|c|c|c|}
\hline Modelos & & & & \multicolumn{4}{|c|}{ Equação } \\
\hline Langmuir & & & & \multicolumn{4}{|c|}{$\mathrm{Qe}=\mathrm{KMCe} /(1+\mathrm{KCe})$} \\
\hline Langmuir - Linear & & & & \multicolumn{4}{|c|}{$\mathrm{Ce} / \mathrm{Qe}=\mathrm{C} / \mathrm{M}+1 /(\mathrm{KM})$} \\
\hline Freundlich & & & & \multicolumn{4}{|c|}{$\mathrm{Qe}=\mathrm{KCe}^{\beta}$} \\
\hline Freundlich - Linear & & & & \multicolumn{4}{|c|}{$\log (\mathrm{Qe})=\log (\mathrm{K})+\beta \log (\mathrm{Ce})$} \\
\hline Langmuir - Freundlich & & & & \multicolumn{4}{|c|}{$\mathrm{Qe}=\mathrm{KMCe}^{\beta} /\left(1+\mathrm{KCe}^{\beta}\right)$} \\
\hline Modelos & M & Erro Padrão & $\mathrm{K}$ & Erro padrão & $\beta$ & Erro padrão & $\mathrm{R}^{2}$ \\
\hline Langmuir & 26,856 & 5,605 & 0,183 & 0,060 & - & - & $92,01 \%$ \\
\hline Langmuir - Linear & 37,907 & 15,860 & 0,101 & 0,011 & - & - & $32,24 \%$ \\
\hline Freundlich & - & - & 4,288 & 0,510 & 0,666 & 0,0855 & $87,98 \%$ \\
\hline Freundlich - Linear & - & - & 3,385 & 1,066 & 0,911 & 0,0611 & $94,88 \%$ \\
\hline Langmuir - Freundlich & 14,667 & 0,891 & 0,243 & 0,029 & 2,347 & 0,2987 & $97,91 \%$ \\
\hline
\end{tabular}

$\mathrm{M}=$ capacidade máxima de adsorção $\left(\mathrm{mg} \mathrm{g}^{-1}\right) ; \mathrm{K}=$ é um parâmetro de afinidade e $\beta=$ é um parâmetro empírico que varia com o grau de heterogeneidade da superfície do adsorvente 


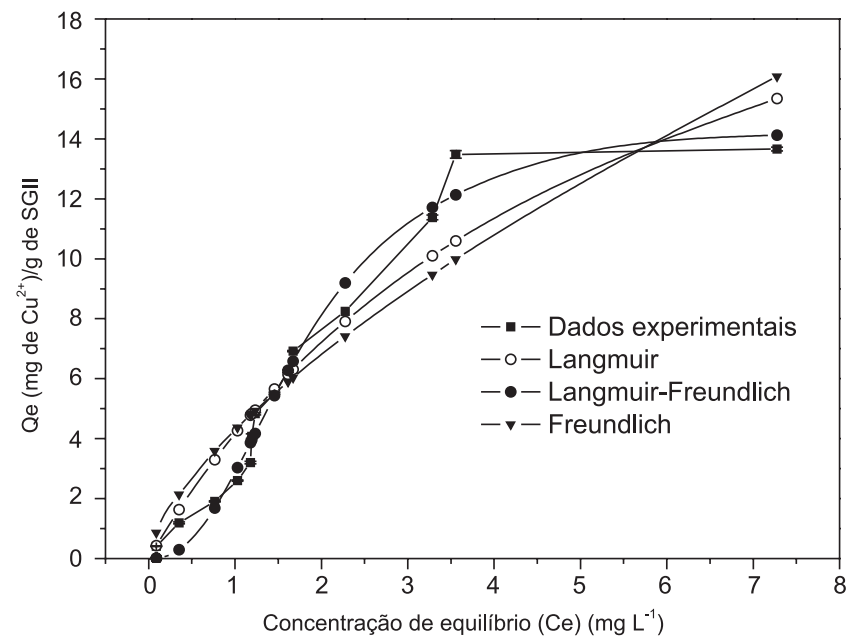

Figura 5. Isoterma de adsorção de $\mathrm{Cu}^{2+}$ na SGII e ajuste dos dados empregando isotermas de Langmuir, Freundlich e Langmuir-Freundlich

de seletividade $(k)$ e do coeficiente de seletividade relativo $\left(k^{\prime}\right)$. Foram observados maiores valores de $\mathrm{K}_{\mathrm{d}}$ para $\mathrm{Cu}^{2+}$ nas misturas binárias $\mathrm{Cu}^{2+} / \mathrm{Ni}^{2+}$ e $\mathrm{Cu}^{2+} / \mathrm{Pb}^{2+}$ para todos os adsorventes avaliados, com predominância para a SGII. Estes dados refletem em elevados valores de $k, 69,40$ e 32,12, respectivamente, para as misturas $\mathrm{Cu}^{2+}$ / $\mathrm{Ni}^{2+}$ e $\mathrm{Cu}^{2+} / \mathrm{Pb}^{2+}$ na SGII. Apesar dos parâmetros $\mathrm{K}_{\mathrm{d}}$ e $k$ indicarem maior adsorção de íons $\mathrm{Cu}^{2+}$ na SGII em detrimento aos íons $\mathrm{Ni}^{2+} \mathrm{e}$ $\mathrm{Pb}^{2+}$, estes não fornecem informações seguras acerca da seletividade do adsorvente, tendo em vista que a maior adsorção dos íons $\mathrm{Cu}^{2+}$ na SGII pode ser decorrente de interações não específicas. Portanto, o emprego do parâmetro $k$ ', por relacionar o valor de $k$ para o adsorvente impresso com aqueles dos adsorventes controle (SGSI e SG), permite obter melhor estimativa do efeito de impressão da SGII para os íons $\mathrm{Cu}^{2+}$. Assim sendo, analisando-se os parâmetros $k^{\prime}$ observa-se que o efeito de impressão da SGII para o sistema $\mathrm{Cu}^{2+} / \mathrm{Ni}^{2+}$ é 6,84 e 5,43 vezes maior do que a SGSI e SG, respectivamente, enquanto que para o sistema $\mathrm{Cu}^{2+} / \mathrm{Pb}^{2+}$ os resultados foram 6,64 e 19,83.

Embora o efeito de impressão tenha sido obtido na SGII, do ponto de vista prático se faz necessário avaliar o comportamento da adsorção de $\mathrm{Cu}^{2+}$ na presença de outros íons metálicos, uma vez que no método desenvolvido, a detecção do analito ocorre via espectrofotometria após reação com DDTC, um complexante não específico para íons $\mathrm{Cu}^{2+}$. Os íons de estudo foram $\mathrm{Ni}^{2+}, \mathrm{Cd}^{2+}, \mathrm{Zn}^{2+}, \mathrm{Co}^{2+}, \mathrm{Ca}^{2+}, \mathrm{Sb}^{3+}, \mathrm{Hg}^{2+}, \mathrm{Pb}^{2+}, \mathrm{Fe}^{2+}$, avaliados nas seguintes proporções (analito:interferente) $1: 1 ; 1: 10 \mathrm{e}$ 1:100. Este estudo foi realizado pré-concentrando $14,0 \mathrm{~mL}$ de uma solu- ção binária contendo $\mathrm{Cu}^{2+}$ na concentração de $250 \mu \mathrm{g} \mathrm{L}{ }^{-1}$. Adotou-se como interferência na determinação do cobre um percentual de recuperação do sinal analítico menor que $90 \%$ ou maior que $110 \%$. Foram observadas interferências negativas para os íons $\mathrm{Ni}^{2+}, \mathrm{Sb}^{3+}, \mathrm{Pb}^{2+} \mathrm{Co}^{2+}, \mathrm{Hg}^{2+} \mathrm{e} \mathrm{Fe}^{2+}$ apenas na concentração de $25,0 \mathrm{mg} \mathrm{L}^{-1}$, ou seja, 100 vezes maior que os íons $\mathrm{Cu}^{2+}$. Esta observação é resultado de uma competição entre o analito e o interferente pelos sítios da SGII. Apesar desta interferência, cabe ressaltar que as concentrações destes íons metálicos em amostras de água são substancialmente menores e, portanto, como será demonstrado não haverá interferência durante a aplicação do método em amostras reais.

\section{Figuras de mérito e aplicação do método em amostras de água}

O desempenho analítico do método foi avaliado por meio das seguintes figuras de mérito: faixa linear da curva analítica de calibração, limites de detecção e quantificação, precisão em termos de repetibilidade e exatidão. O desempenho do sistema de pré-concentração foi avaliado com base no fator de pré-concentração (PFC), eficiência de concentração (EC) e índice de consumo (IC).

A curva analítica feita com a etapa de pré-concentração de cobre apresentou linearidade satisfatória $(r=0,995)$ dentro da faixa de concentração de 30,0 a $300,0 \mu \mathrm{g} \mathrm{L^{-1 }}$ com a equação linear Abs $=-0,0533+0,0042\left[\mathrm{Cu}^{+2}\left(\mu \mathrm{g} \mathrm{L^{-1 }}\right)\right]$. A curva analítica sem o uso da etapa de extração foi $\mathrm{Abs}=-0,01671+0,000135\left[\mathrm{Cu}^{+2}\left(\mu \mathrm{g} \mathrm{L}^{-1}\right)\right] \mathrm{com}$ coeficiente de correlação de 0,99687 utilizando padrões de 500,0 a $3000,0 \mu \mathrm{g} \mathrm{L}^{-1}$. O fator de pré-concentração (FPC), calculado pela razão entre o coeficiente angular da curva feita com e sem a etapa de pré-concentração foi de 31,1 vezes, rendendo limites de detecção (LD) e quantificação (LQ) de 3,4 e $11,4 \mu \mathrm{g} \mathrm{L}^{-1}$, respectivamente. ${ }^{32}$ No que concerne à precisão $(n=7)$ a análise de padrões de cobre nas concentrações de 20,0 e $280,0 \mu \mathrm{g} \mathrm{L}^{-1}$ rendeu desvios padrão relativos (DPR) de 3,21 e 1,21\%, respectivamente.

A eficiência de concentração expressa o fator de pré-concentração obtido durante 1 min de pré-concentração. Portanto, como a etapa de pré-concentração requereu 1 min e $22 \mathrm{~s}$, a EC obtida foi de 22,75 $\mathrm{min}^{-1}$. O índice de consumo (IC) estabelece o volume de amostra, em mililitros, necessário para obter um valor unitário de FPC. Assim sendo, como a etapa de pré-concentração foi realizada numa vazão de 10,2 $\mathrm{mL} \min ^{-1}$ durante 1 min e $22 \mathrm{~s}$, rendendo um fator de pré-concentração de 31,1, o valor do IC torna-se 0,45 mL. Finalmente, a frequência de amostragem, que reflete a quantidade de amostras lidas por hora, foi de $32 \mathrm{~h}^{-1}$. Uma comparação do método proposto com outros dedicados à determinação de cobre por espectrofotometria após etapa de préconcentração em fase sólida permite destacar como vantagens o baixo consumo de amostra, reduzido limite de detecção, ausência de complexantes no sistema em fluxo e elevada frequência analítica (Tabela 3 ).

Tabela 2. Coeficiente de distribuição, seletividade e seletividade relativa para os íons $\mathrm{Cu}^{2+}$ e $\mathrm{Ni}^{2+}$ nos adsorventes SGII, SGSI e SG

\begin{tabular}{|c|c|c|c|c|c|c|c|}
\hline \multirow[t]{2}{*}{ Adsorventes } & \multicolumn{2}{|c|}{$\begin{array}{l}\text { Concentração inicial } \\
\qquad\left(\mathrm{mg} \mathrm{L}^{-1}\right)\end{array}$} & \multicolumn{2}{|c|}{$\mathrm{K}_{\mathrm{d}}\left(\mathrm{mL} \mathrm{g}^{-1}\right)$} & \multirow[t]{2}{*}{$k$} & \multicolumn{2}{|c|}{$k^{\prime}$} \\
\hline & $\mathrm{Cu}^{2+}$ & $\mathrm{Ni}^{2+}$ & $\mathrm{Cu}^{2+}$ & $\mathrm{Ni}^{2+}$ & & SGII/SGSI & SGII/SG \\
\hline SGII & 2 & 2 & 4967 & 71,57 & 69,40 & & \\
\hline SGSI & 2 & 2 & 836,8 & 82,53 & 10,14 & 6,84 & 5,43 \\
\hline \multirow[t]{3}{*}{ SG } & 2 & 2 & 3190 & 249,5 & 12,78 & & \\
\hline & \multicolumn{2}{|c|}{$\begin{array}{l}\text { Concentração inicial } \\
\qquad\left(\mathrm{mg} \mathrm{L}^{-1}\right)\end{array}$} & \multicolumn{2}{|c|}{$\mathrm{K}_{\mathrm{d}}\left(\mathrm{mL} \mathrm{g}^{-1}\right)$} & $k$ & \multicolumn{2}{|c|}{$k^{\prime}$} \\
\hline & $\mathrm{Cu}^{2+}$ & $\mathrm{Pb}^{2+}$ & $\mathrm{Cu}^{2+}$ & $\mathrm{Pb}^{2+}$ & & SGII/SGSI & SGII/SG \\
\hline SGII & 4 & 4 & 86600 & 2696 & 32,12 & & \\
\hline SGSI & 4 & 4 & 1824 & 377,2 & 4,84 & 6,64 & 19,83 \\
\hline SG & 4 & 4 & 5043 & 3105 & 1,62 & & \\
\hline
\end{tabular}


Tabela 3. Comparação de diferentes métodos de pré-concentração em fase sólida com detecção espectrofotométrica para determinação de cobre

\begin{tabular}{|c|c|c|c|c|c|c|}
\hline Adsorvente & Agente complexante & $\begin{array}{l}\text { Modalidade de pré-con- } \\
\text { centração }\end{array}$ & Eluente & $\mathrm{VP}(\mathrm{mL})$ & $\begin{array}{c}\mathrm{LD} \\
\left(\mu \mathrm{g} \mathrm{L} \mathrm{L}^{-1}\right)\end{array}$ & Ref. \\
\hline $\mathrm{C}_{18}$ & Neocuproina & Off-line & Álcool isopentílico & 500 & 0,12 & 33 \\
\hline DAPPS & DDTC & On-line & $\mathrm{HNO}_{3}$ & 13 & 8,4 & 34 \\
\hline Resina Lewatit TP807'84 & Sulfasazina & On-line & $\mathrm{HCl}$ & 100 & 2,0 & 35 \\
\hline Benzofenona Microcristalina & Cupferrona & Off-line & Etanol & 100 & 5,5 & 36 \\
\hline SGII & DDTC & On-line & $\mathrm{HNO}_{3}$ & 14 & 3,4 & Este trabalho \\
\hline
\end{tabular}

$\mathrm{VP}=$ volume de pré-concentração; LD = limite de detecção; DAPPS = sílica gel funcionalizada com 3-propil-1,2-diaminopropano; Lewatit TP807’84 resina = resina contendo grupo ácido bis( $2,4,4$ trimetilpentil) fosfínico

Tabela 4. Resultados do teste de exatidão para a determinação de cobre em amostras de água

\begin{tabular}{|c|c|c|c|}
\hline Amostras & $\begin{array}{l}\text { Concentração de cobre } \\
\text { adicionada }\left(\mu \mathrm{g} \mathrm{L} \mathrm{L}^{1}\right)\end{array}$ & $\begin{array}{l}\text { Concentração de cobre } \\
\text { encontrada }\left(\mu \mathrm{g} \mathrm{L}^{-1}\right)\end{array}$ & $\begin{array}{c}\text { Recuperação } \\
(\%)\end{array}$ \\
\hline \multirow[t]{2}{*}{ Água do Lago de Furnas (1) } & 0 & $36,5 \pm 1,67$ & - \\
\hline & 30,0 & $68,6 \pm 1,15$ & 103,2 \\
\hline \multirow[t]{2}{*}{ Água do Lago de Furnas (2) } & 0 & $39,2 \pm 1,6$ & \\
\hline & 40,0 & $80,8 \pm 3,0$ & 102,0 \\
\hline \multirow[t]{2}{*}{ Água do Lago de Furnas (3) } & 0 & $37,2 \pm 1,9$ & \\
\hline & 40,0 & $78,3 \pm 1,9$ & 101,0 \\
\hline \multirow[t]{2}{*}{ Água de Mar (simulada) } & 0 & $<\mathrm{LD}$ & - \\
\hline & 30,0 & $27,6 \pm 1,3$ & 92,0 \\
\hline \multirow[t]{2}{*}{ Água potável } & 0 & $<\mathrm{LD}$ & - \\
\hline & 30,0 & $29,7 \pm 0,3$ & 99,0 \\
\hline \multirow[t]{2}{*}{ Água mineral - marca A } & 0 & $<\mathrm{LD}$ & - \\
\hline & 30,0 & $30,0 \pm 1,2$ & 100,0 \\
\hline \multirow[t]{2}{*}{ Água mineral - marca B } & 0 & $<\mathrm{LD}$ & - \\
\hline & 30,0 & $29,5 \pm 1,7$ & 98,5 \\
\hline
\end{tabular}

LD = limite de detecção. (1) = ponto de coleta - Rio Sapucaí, (2) = Amostra coletada no município de Fama (junção dos Rios Sapucaí e Machado), (3) = Rio São Thomé. Os resultados são expressos como média \pm desvio padrão. (n=3)

A exatidão do método foi avaliada por meio de testes de adição e recuperação (Tabela 4) com emprego de amostras de água de diferentes composições. Amostras de água do Lago de Furnas, potável, mineral e amostras de água de mar simulada foram enriquecidas com quantidades conhecidas de cobre, com posterior ajuste do $\mathrm{pH}$ com solução tampão amoniacal. A composição da água de mar simulada foi baseada de acordo com a literatura, ${ }^{37}$ sendo de $27,9 \mathrm{~g} \mathrm{~L}^{-1}$ de $\mathrm{NaCl}$; 1,4 $\mathrm{g} \mathrm{L}^{-1}$ de $\mathrm{KCl} ; 2,8 \mathrm{~g} \mathrm{~L}^{-1}$ de $\mathrm{MgCl}_{2} ; 0,5 \mathrm{~g} \mathrm{~L}^{-1}$ de $\mathrm{NaBr}$ e $2,0 \mathrm{~g} \mathrm{~L}^{-1}$ de $\mathrm{MgSO}_{4}$. As amostras do Lago de Furnas foram coletadas em frascos de polietileno, seguidas da adição de $3 \mathrm{~mL}$ de $\mathrm{HNO}_{3}$ concentrado para cada $100 \mathrm{~mL}$ de amostra coletada e estocadas em freezer até o uso. As amostras foram filtradas em membranas de acetato de celulose 0,45 $\mu \mathrm{m}$ sob vácuo e o pH ajustado com tampão amoniacal.

Como pode ser constatada, a exatidão dos métodos é assegurada para todas as amostras de água analisadas com base nos percentuais de recuperação $(92,0$ a 103,2\%).

\section{CONCLUSÃO}

No presente trabalho foi descrito que a sílica gel funcionalizada com 2-aminoetil-3-aminobutilmetildimetoxissilano e com impressão iônica apresenta seletividade superior para os íons $\mathrm{Cu}^{2+}$ em comparação com os adsorventes controle (SGSI e SG), mesmo na presença de interferentes metálicos em potencial $\left(\mathrm{Ni}^{2+} \mathrm{e} \mathrm{Pb}^{2+}\right)$. Adicionalmente, o adsorvente com impressão iônica bidimensional não apresenta apenas como vantagem a seletividade, mas também maior capacidade adsortiva em relação a outros adsorventes impressos preparados por métodos com impressão tridimencional.

Mediante as características satisfatórias da SGII, desenvolveu-se um método analítico de pré-concentração on-line de íons cobre com detecção espectrofotométrica que exibiu satisfatório fator de enriquecimento $(31,1)$ e baixo limite de detecção $\left(3,43 \mu \mathrm{g} \mathrm{L}^{-1}\right)$. Uma característica da SGII que deve ser ressaltada é a ausência de elevada pressão no interior da minicoluna, mesmo trabalhando com vazões elevadas $(10,2$ $\mathrm{mL} \min ^{-1}$ ), o que confere uma frequência analítica elevada ao método.

Finalmente, os resultados obtidos neste trabalho evidenciam que a técnica de impressão iônica dimensional sobre suportes sólidos, associada ao uso de novos organossilanos, se mostra promissora para a concepção de métodos de pré-concentração de íons metálicos visando o aumento de sensibilidade e seletividade de técnicas analíticas, em especial a espectrofotometria UV-vis.

\section{AGRADECIMENTOS}

À FAPEMIG (Fundação de Amparo à Pesquisa do Estado de Minas Gerais), CNPq (Conselho Nacional de Desenvolvimento Cien- 
tífico e Tecnológico), CAPES (Coordenação de Aperfeiçoamento de Pessoal de Nível Superior) e INCT (Instituto Nacional de Ciência e Tecnologia) de Bioanalítica (processo n 57.3672/2008-3) pelo auxílio financeiro. C. R. T. Tarley agradece ao CNPq pela concessão da bolsa produtividade científica.

\section{REFERÊNCIAS}

1. Namiésnik, J.; Crit. Rev. Anal. Chem. 2000, 20, 22.

2. Vandecasteele, C. V.: Block, C. B.; Modern Methods for Trace Element Determination, John Wiley \& Sons: New York, 1993.

3. Tarley, R. T. T.; Ferreira, S. L. C.; Arruda, M. A. Z.; Microchem. J. 2004, $77,163$.

4. Pereira, M. G.; Arruda, M. A. Z.; Microchim. Acta 2003, 141, 115.

5. Tarley, C. R. T.; Arruda, M. A. Z.; Anal. Sci. 2005, 20, 961.

6. Pauling, L. J.; J. Am. Chem. Soc. 1940, 62, 2643.

7. Wulff, G.; Sarhan, A.; Angew. Chem., Int. Ed. 1972, 11, 341.

8. Nishide, H.; Deguchi, J.; Chem. Lett. 1976, 5, 69.

9. Rao, T. P.; Kala, R.; Daniel, S.; Anal. Chim. Acta 2006, 578, 105.

10. Birlik, E.; Anal. Chim. Acta 2006, 565, 145.

11. Tarley, C. R. T.; Sotomayor, M. D. T.; Kubota, L. T.; Quim. Nova 2005, 28, 1076

12. Bi, X.; Lau, R. J.; Yang, K.; Langmuir 2007, 23, 8079.

13. Jiang, N.; Chang, X.; Zheng, H.; He, Q.; Hu, Z.; Anal. Chim. Acta 2006, $577,225$.

14. Zheng, H.; Geng, T. M.; Hu, L. M.; Chem. Anal. (Warsaw, Pol.) 2008, $53,673$.

15. Chang, X.; Wang, X.; Jiang, N.; He, Q.; Zhai, Y.; Zhu, X.; Hu, Z.; Microchim. Acta 2008, 162, 113.

16. He, Q.; Chang, X. J.; Zheng, H.; Jiang, N.; Wang, X.; Int. J. Environ. Anal. Chem. 2008, 88, 373.

17. Zhang, N.; Hu, B.; Hung, C. Z.; Anal. Chim. Acta 2007, 597, 12.

18. Zheng, H.; Zhang, D.; Wang, W. Y.; Fan, Y. Q.; Han, H. P.; Microchem. Acta 2007, 157, 7 .
19. Chang, X. J.; Jiang, N.; Zheng, H.; He, Q.; Hu, Z.; Zhai, Y.; Cui, Y.; Talanta 2007, 71, 38.

20. He, Q.; Chang, X. J.; Zheng, H.; Jiang, N.; Hu, Z.; Zhai, Y.; Chem. Anal. (Warsaw, Pol.) 2006, 51, 715.

21. Reis, B. F.; Filho, H. B.; Quim. Nova 1993, 16, 570.

22. Andaçu, M.; Ozyapi, E.; Senel, S.; Say, R.; Denizli, A.; Ind. Eng. Chem. Res. 2006, 45, 1780.

23. Wang, X. G.; Lin, K. S. K.; Chan, J. C. C.; Cheng, S.; J. Phys. Chem. B 2005, 109, 1763.

24. Finnie, K. S.; Thompson, J. G.; Withers, R. L.; J. Phys. Chem. Solids 1994, 55, 23

25. Qu, R.; Wang, M.; Sun, C.; Chunnuan, Y. Z.; Chen, H.; Meng, Y.; Yin, P.; Appl. Surf. Sci. 2008, 255, 3361.

26. Skoog, D. A.; Holler, F. J.; Nieman T. A.; Principles of Instrumental Analysis, $5^{\text {th }}$ ed., Harcout College Publishers: San Francisco, 1998.

27. Gilles, C. H.; Smith. A.; J. Colloid Interface Sci. 1974, 47, 755.

28. Airoldi, C.; Farias, R. F.; Quim. Nova 2000, 23, 496

29. Shamsipur, M.; Fasihi, J.; Khanchi, A.; Hassani, R.; Alizadeh, K.; Shamsipur, H.; Anal. Chim. Acta 2007, 599, 294.

30. Liu, Y.; Chang, X.; Wang, S.; Guo, Y.; Din, B.; Meng, S.; Anal. Chim. Acta 2004, 519, 173.

31. Umpleby, R. J.; Baxter, S. C.; Rampey, A. M.; Rushton, G. T.; Chen, Y.; Shimizu, K. D.; J. Chromatogr., B 2004, 804, 141.

32. Long, G. L.; Winefordner, J. D.; Anal. Chem. 1983, 55, 712.

33. Yamini, Y.; Tamaddon, A.; Talanta 1999, 49, 119.

34. Moraes, S. V. M.; Brasil, J. L.; Milcharek, C. D.; Martins, L. C.; Laranjo, T.; Gallas, M. R.; Benvenutti, E. V.; Lima, E. C.; Spectrochim. Acta, Part A 2005, 62, 398.

35. Castilho, E.; Cortina, J. L.; Beltrán, J.; Prat, M.; Granados, M.; Analyst 2001, 126, 1149 .

36. Lee, T.; Choi, H.; Bull. Korean Chem. Soc. 2002, 23, 861.

37. Zougagh, M.; Rudner, P. C.; Torres, A. G.; Pavon, J. M. C.; J. Anal. At. Spectrom. 2000, 15, 1589. 